\title{
Infant-Directed Speech Becomes Less Redundant as Infants Grow: Implications for Language Learning
}

\author{
Shira Tal ${ }^{1,2}$, Eitan Grossman ${ }^{3}$ and Inbal Arnon ${ }^{4}$ \\ ${ }^{1}$ Department of Linguistics and English Language, University of Edinburgh \\ ${ }^{2}$ Department of Cognitive Sciences, The Hebrew University of Jerusalem \\ 3 Department of Linguistics, The Hebrew University of Jerusalem \\ 4 Department of Psychology, The Hebrew University of Jerusalem
}

\begin{abstract}
Author Note
Correspondence concerning this article should be addressed to Shira Tal, Centre for Language Evolution, School of Philosophy, Psychology and Language Sciences, University of Edinburgh, 3 Charles Street, Edinburgh, EH8 9AD, United Kingdom. E-mail address: stal@ed.ac.uk
\end{abstract}

\section{Supplementary material}

https://osf.io/kj6mg/?view_only=97ofcc9fc4fo4940a2821b74eo31e51c 


\begin{abstract}
According to the communicative efficiency hypothesis, speakers should increase redundancy when they perceive difficulties in comprehension. This effect was mostly studied by looking at the impact of message complexity and predictability, with less work looking at the impact of interlocutors' perceived difficulty. Here, we ask whether conversing with language learners results in more redundant language. We test this by quantifying redundancy in infant-directed speech using entropy rate - an information-theoretic measure reflecting average degree of repetitiveness. While IDS is often described as repetitive, entropy rate provides a novel holistic measure of redundancy in this speech genre. Using two developmental corpora, we compare entropy rates of samples taken from different ages. We find that parents use less redundant speech when talking to older infants, illustrating an effect of interlocutor proficiency on redundancy. Interestingly, the developmental decrease in redundancy is largely driven by a decrease in repetitions of multi-word sequences, highlighting the importance of larger sequences in early language learning.
\end{abstract}

Keywords: infant-directed speech, entropy rate, efficient communication, multi-word sequences, redundancy 


\section{Infant-Directed Speech Becomes Less Redundant as Infants Grow: Implications for Language Learning}

Speakers have different ways to express the same (or roughly the same) message, using more or less linguistic material. For example, they can select between different constructions, e.g., "Susan gave toys to the children" vs. "Susan gave the children toys" (Bresnan, Cuetni, Nikitiana, \& Baayen, 2007); they can articulate words faster or slower (Aylett \& Turk, 2004); they can use or omit optional syntactic elements, e.g., "I saw that Steve ran a marathon" vs. "I saw Steve ran a marathon" (Ferreira, 2008); and they can refer to the same entities using more or fewer words, e.g., "she", vs. "a good friend of mine" (Gundel, Hedberg, \& Zacharski, 1993). The factors guiding speakers' choices have been the focus of much psycholinguistic research, as a way to investigate the impact of competing pressures on language use (for a review see Jaeger \& Buz, 2017). One explanation for speaker behavior is that language users are biased for efficient communication, so that their choices reflect a trade-off between the two competing pressures of reducing effort and maximizing understandability (Grice, 1975; Piantadosi et al., 2011b; Zipf, 1949). On the one hand, speakers aim to maximize understandability by providing enough information to successfully transmit their message. At the same time, speakers aim to minimize production effort, by exerting as little effort as possible.

An efficient way of balancing these pressures is to produce less linguistic material for more predictable messages: assigning longer signals to less expected messages and shorter signals to more expected messages can facilitate listener comprehension and balance the overall information rate of the interaction (Aylett \& Turk, 2004; Levy \& Jaeger, 2007; Pate \& Goldwater, 2015; Shannon, 1948; Zipf, 1949). In line with this view, there is abundant evidence that speakers tend to reduce or omit more predictable elements, at many levels of linguistic analysis (syllable, word, construction: Aylett \& Turk, 2004; Cohen Priva, 2015; Frank \& Jaeger, 2008; Jaeger, 2010; Kravtchenko, 2014; Kurumada \& Jaeger, 2015; Levy \& Jaeger, 2007; Mahowald, Fedorenko, 
Piantadosi, \& Gibson, 2013; Pate \& Goldwater, 2015; Piantadosi et al., 2011; Tily \& Piantadosi, 2009). For example, when choosing between shorter and longer lexical forms with the same meaning (e.g., undergrad and undergraduate), speakers tend to use the shorter forms in contexts where they are more predictable (Mahowald et al., 2013). In information-theoretic terms, speakers aim to minimize redundancy in their speech: when possible, they use fewer or shorter elements to convey a more predictable message.

According to the principles of efficient communication, the balance between effort and understandability can change depending on the comprehension difficulty within a conversation. Speakers are predicted to produce more effortful signals (i.e., ones containing more linguistic material) when understandability is at risk. Theoretically, understandability can be impacted by different factors (see discussion in Pate \& Goldwater, 2015): predictability of the linguistic message itself, properties of the environment (e.g., noisy vs. quiet), and characteristics of the interlocutor. Indeed, a great volume of studies demonstrate that speakers are more likely to speak redundantly (i.e., increase effort) when the conveyed meaning is unpredictable in context (Aylett \& Turk, 2004; Kurumada \& Jaeger, 2015; Levy \& Jaeger, 2007; Mahowald et al., 2013). For example, Japanese speakers are more likely to produce optional case marking when the thematic assignment is less predictable in context (e.g., the criminal arrested the police officer vs. the police officer arrested the criminal, Kurumada \& Jaeger, 2015). Other studies document an effect of environmental noise on redundancy: talkers increase effort in the presence of background noise (a phenomenon known as The Lombard Effect, Lombard, 1911; Summers, Pisoni, Bernacki, Pedlow, \& Stokes, Michael, 1988; Zhao \& Jurafsky, 2009).

Finally, another potential source of comprehension difficulty is the interlocutor herself, who could face (or be perceived as facing) difficulty in comprehension, leading the speaker to increase effort. Speakers are perceptive of their audience's knowledge and modify their speech accordingly, a phenomenon known as audience design (Ariel, 1990; Arnold, 2008; Brennan \& Hanna, 2009; Chafe, 1994; Clark \& Murphy, 1983; Heller et al., 2012; Isaacs \& Clark, 1987; 
Lockridge \& Brennan, 2002). Audience design predicts that speakers will modify their speech based on the presumed properties of interlocutors. From the perspective of communicative efficiency, this modification should lead speakers to increase redundancy (increase effort) when their interlocutors misunderstand them (see also Grice, 1975). Indeed, several studies demonstrate this pattern (Buz et al., 2016; Lockridge \& Brennan, 2002; Roche et al., 2013). For example, speakers tend to hyper-articulate potentially confusable words when feedback from their interlocutors suggests that they have misunderstood previous words (Buz et al., 2016). Importantly, speakers can modify their speech not only on the basis of local misunderstandings, but also based on global estimations of their interlocutors' knowledge state and comprehension ease (Arnold, 2008). For example, New Yorkers refer to landmarks differently when talking to other New Yorkers compared to people from other cities (Isaacs \& Clark, 1987). While global interlocutor properties can impact overall comprehension difficulty, their impact on speaker effort has been relatively under-studied.

In this paper we use infant-directed speech as a case study to explore the way speakers modify the amount of redundancy in their speech based on global properties of their interlocutors, and specifically on their level of linguistic proficiency. If speakers use more linguistic material with interlocutors that are perceived to have more comprehension difficulty, then speech directed to young infants, who are in the early stages of language learning, should be more redundant than speech directed to older children. This idea is supported by several lines of converging evidence. First, recent studies have found that adult speakers provide more redundant acoustic signals when talking to infants compared to proficient adult speakers (Pate \& Goldwater, 2015; Tippenhauer et al., 2020; Uther et al., 2007). Adults reduced the phonetic duration of more predictable words to a smaller extent when talking to infants compared to adults (Pate \& Goldwater, 2015; Tippenhauer et al., 2020), and hyperarticulated vowels (provided more acoustic information in the vowel formants) more (Uther et al., 2007). More generally, infant-directed speech (IDS) differs from adult-directed speech (ADS) in many respects (for reviews see Schwab 
\& Lew-Williams, 2016; Soderstrom, 2007), and has several prominent characteristics which could make it more redundant. Among other properties, IDS is characterized by lower lexical diversity (Soderstrom, 2007), frequently recurring phrases (Cameron-Faulkner et al., 2003), and successive utterances with partial self-repetitions (often called variation sets, Küntay \& Slobin, 1996; Tal \& Arnon, 2018; Waterfall, 2006).

In the current study we investigate developmental changes in redundancy in IDS, with two goals in mind: (1) Providing a novel measure of global redundancy in IDS, and (2) Asking whether redundancy is impacted by the interlocutors' perceived overall proficiency level, a core prediction of the communicative efficiency hypothesis. While infant-directed speech is often described as repetitive, this is usually based on the analysis of individual properties (e.g., lexical types, frequent frames, variation sets), rather than on a holistic evaluation. Here, we use entropy rate, an information-theoretic measure that allows us to capture the redundancy of a text as a whole (see details below). If speakers use more linguistic material with less proficient speakers, then speech directed to infants should decrease in redundancy with development. We investigate this hypothesis in two corpus studies. In Study 1 we use two developmental corpora to ask whether redundancy in IDS decreases with age. Having found an effect of age on redundancy, in Study 2 we ask which property of the input drives this effect. Specifically, we ask whether the increased redundancy in speech directed to younger infants is the result of a more repetitive lexicon or more repetitive multi-word sequences. While it is known that lexical diversity increases with age, less is known about the proportion of recurring multi-word sequences. Such sequences, however, are a salient property of IDS (Cameron-Faulkner et al., 2003; Goldberg, 2019) and have been shown to play an important role in learning various grammatical relations (Arnon, 2021; Arnon \& Clark, 2011; Arnon \& Ramscar, 2012; Siegelman \& Arnon, 2015). Finding that increased redundancy in young children's input is (also) driven by the repetition of multi-word sequences will provide additional evidence for their role in early learning. 


\section{Quantifying redundancy using entropy measures}

Recent years have seen increased interest in the application of information-theoretic concepts and methods to the study of language (e.g., Bentz, Alikaniotis, Cysouw, \& Ferrer-i-Cancho, 2017; Cohen Priva, 2015; Ferdinand, Kirby, \& Smith, 2019; Juola, 20o8; Piantadosi, Tily, \& Gibson, 2011; Zaslavsky, Kemp, Regier, \& Tishby, 2018). These studies have provided novel insights into long-debated questions about language structure, learning, and use (see review in Gibson et al., 2019). Information-theoretic measures such as entropy (Shannon, 1948) quantify the amount of uncertainty in a distribution of linguistic elements and have been shown to impact real-time language processing (Jaeger, 2010; Linzen \& Jaeger, 2015; Piantadosi et al., 2011a; Siegelman et al., 2020). Such measures can also be used to characterize language systems as a whole. For example, information-theoretic measures can be used to measure and compare the complexity of the same text across languages, with texts with lower entropy seen as less complex (Bentz et al., 2017, 2016; Ehret, 2018; Ehret \& Szmrecsanyi, 2016, 2019; Juola, 2008; Koplenig et al., 2017; Lupyan, 2019). One particularly relevant measure for our purposes is entropy rate, which quantifies redundancy in texts by calculating their average degree of repetitiveness. Unlike unigram entropy, entropy rate does not treat words independently and captures the sequential relation between words and the degree of repetitiveness in a text (for a discussion see Bentz et al., 2017). Importantly, entropy rate provides us a way to assess redundancy in texts overall, without having to pre-suppose where that redundancy comes from (bigrams, trigrams, lexical repetitions, etc.). As such, it provides a more comprehensive measure of redundancy than merely looking at repetitions of a particular size. Another advantage of entropy rate is that it has been used previously to compare redundancy across different languages. Using this measure therefore allows us to tie together the literature on infant-directed speech and the typological literature. In particular, this measure was used in a recent study (Bentz et al., 2017) to compare translations of the same text in over 1200 languages. Entropy rate was found to be similar across languages, suggesting that languages show similar levels of complexity. Interestingly, no study to date has 
used entropy rate to examine redundancy across language registers within the same language. Here, we will use entropy rate as a way to quantify redundancy in IDS, and ask whether it changes with development. In what follows we explain how entropy rate can be used to quantify redundancy.

The entropy rate of words provides the average information content of words conditioned on all preceding tokens. Entropy rate is affected by the order of words in a given text: it decreases when words are more predictable given their preceding contexts. Entropy rate is calculated in the following way (see Eq. 1): for any given word $^{1}$ in the text, we look for the longest string it initiates that is found in the preceding text. For example, the longest string found for the underlined the in the sequence 'The girl saw the girl next door', will be the the girl, as the repetition of the alone is shorter than the repetition of the girl (see more details below).

(1) $\mathrm{h}(\mathrm{T})=\frac{1}{N} \sum_{i=2}^{N} \frac{\log _{2} i}{L i}$

The entropy rate is the average of these match lengths where $\mathrm{N}$ is the overall number of tokens, $\mathrm{i}$ is the position in the string, and $\mathrm{Li}$ is the longest match string for $\mathrm{i}$ plus one ( $\mathrm{Li}=1+$ the longest match string). The average length of these strings reflects the redundancy in the text: the larger the average match string is, the more repetitive (and therefore, redundant) the text is. Mathematically, as the average match string grows, entropy rate becomes smaller. In other words, a lower entropy rate reflects a more redundant text. To give an example, we use the following short text (numbers index word order):

(2) come $_{1}$ here $_{2}$ come $_{3}$ here $_{4}$

In order to calculate entropy rate for this text, we start with the second token (here). Because there is no match for this word in the preceding string (which contains only the word

${ }^{1} \mathrm{~A}$ word is defined by white spaces, and is therefore identified orthographically by form, not lemma. That means $d o g$ and $\operatorname{dog} s$, for example, are identified as different words. A string is any sequence starting from one word. 
come), the match length for this token is $0 . \mathrm{L} 2$ is $1(1+$ the length of the string match: 0$)$, and the total information content for this word is $\frac{\log _{2} 2}{1+0}=1$. Moving on to the next word $(\mathrm{i}=3)$, come $e_{3}$, we find a match of length 1 in come $e_{1}$. We then expand our search string, going one word forward from the word in question, and look for a match for the sequence come $e_{3} h e r e_{4}$. We find a match for the two-word sequence in the preceding text, meaning that the match length for this token is 2 , and the information content is: $\frac{\log _{2} 3}{1+2}=0.528$. Finally, the longest matching string for the last word, $h_{e r e_{4}}$, is only one word long (here $e_{2}$, and the information content is: $\frac{\log _{2} 4}{1+1}=1$. The total entropy rate for this text is the average information content of all words, which is 0.632 .

To illustrate how this measure captures different types of redundancies, we compare the following three short texts (after removing punctuation marks):

[A] where is the ball look at this banana

[B] where is the ball where is the ball

[C] where ball the where the ball is is

All three texts are composed of eight words. In text [A] all words are unique while Text [B] has only 4 unique words. Importantly, Text [B] is composed of two repetitive four-word sequences, making it more repetitive (i.e., redundant) than text [A]. Text [C] has the exact same words as text [B], but does not include repeated sequences. Text [B] and Text [C] have the same level of lexical repetition (four words, each repeating twice), but differ in the recurrence of multiword sequences: text [C] is less repetitive, and therefore less redundant. Because of these differences, text [A] will have the highest entropy rate (it is the least redundant of the three texts), text [B] will have the lowest entropy rate (it is the most redundant) and text [C] will be somewhere in between. This is indeed the case: $h(A)=1.91, h(B)=1.02, h(C)=1.29$.

Entropy rate provides us with a global measure of redundancy that is sensitive to the order of words; is based on repetitions of varying sizes; and can be used to compare different language registers. In Study 1 we compare entropy rate in samples of IDS directed to different ages. If 
speech to younger infants is more redundant, then entropy rate should increase with age. In Study 2, we explore the relative impact of lexical and sequential repetition on the decrease in redundancy. This investigation is motivated by the language development literature, where there is growing evidence for the importance of multi-word sequences in language acquisition (AbbotSmith \& Tomasello, 2006; Arnon, 2016, 2021; Arnon \& Christiansen, 2017; Schwab \& LewWilliams, 2016b; Stoll et al., 2009). Building on this literature, Study 2 tests the relative contribution of lexical repetition vs. repetition of multi-words sequences to the change in entropy rate.

\section{Study 1}

\section{Method}

\section{The corpora}

We used two developmental corpora, both taken from the CHILDES database (MacWhinney, 2000). The first is the NewmanRatner Corpus (Newman et al., 2016), which contains transcripts of 122 English-speaking mother-child dyads, who were recorded during lab visits as part of a longitudinal study. Each mother and child were recorded for 15 minutes during free play with a box of toys. The recordings were taken at the ages of 7,10,11,18 and 24 months, though not all children took part in all time points. We analyzed only three time points from this corpus (7-, 11- and 24-months), since the rest did not have enough data (see Procedure below). This corpus has data from many dyads all engaged in the same activity (playing with the same box of toys). See Table 1 for a summary of IDS measures of this corpus. Note that unlike the more familiar developmental pattern (Schwab \& Lew-Williams, 2016a; Soderstrom, 2007), type-token ratio does not increase with age in this corpus. We come back to this issue in Study 2.

The second corpus we used is the Providence Corpus (Demuth et al., 2006), accessed using the childesr package in $\mathrm{R}$ (version 2018.1, Sanchez et al., 2019). This corpus contains dense longitudinal recordings of six monolingual English-speaking children between the ages of 1-4 years, collected during naturalistic interactions with their caregivers at home. We analyzed the 
data of the three children that had the most data - Ethan, Lily and Naima. We created two developmental time points by dividing the recordings into two age bins (12-24 months and 24-36 months; beyond this age there was not enough data to match our purposes). Whereas the NewmanRatner Corpus has many dyads recorded under fairly controlled lab conditions, the Providence Corpus has few children recorded across multiple activities. Together these two sets of corpora provide a rich representation of IDS in different contexts. See Table 2 for a summary of IDS measures of this corpus.

\section{Table 1}

Summary of IDS properties in the NewmanRatner Corpus (values represent mean per interaction)

\begin{tabular}{cccc}
\hline Age in months & $\begin{array}{c}\text { Number of } \\
\text { words }\end{array}$ & Type-token ratio & $\begin{array}{c}\text { Mean length of } \\
\text { utterance }\end{array}$ \\
\hline $\mathbf{7}$ & 759.5 & 0.32 & 3.37 \\
$\mathbf{1 1}$ & 759.23 & 0.3 & 3.28 \\
$\mathbf{2 4}$ & 1160.1 & 0.25 & 3.8 \\
\hline
\end{tabular}

\section{Table 2}

Summary of IDS properties in the Providence Corpus (values represent mean per interaction)

\begin{tabular}{ccccc}
\hline Child & $\begin{array}{c}\text { Age in } \\
\text { months }\end{array}$ & $\begin{array}{c}\text { Number of } \\
\text { words }\end{array}$ & $\begin{array}{c}\text { Type-token } \\
\text { ratio }\end{array}$ & $\begin{array}{c}\text { Mean length } \\
\text { of utterance }\end{array}$ \\
\hline Naima & $12-24$ & 4075.2 & 0.17 & 5.25 \\
\multirow{3}{*}{ Lily } & $24-36$ & 3738.1 & 0.19 & 5.66 \\
& $12-24$ & 4606.75 & 0.18 & 4.55 \\
\multirow{3}{*}{ Ethan } & $24-36$ & 4553.51 & 0.2 & 5.65 \\
& $12-24$ & 3066.18 & 0.19 & 4.18 \\
& $24-36$ & 3688.82 & 0.19 & 4.75 \\
\hline
\end{tabular}

\section{Procedure}

We calculated entropy rate using the Hrate package (Bentz et al., 2017) in R (all analyses were conducted using R Version 3.6.1, R Core Team, 2019). Since this is the first developmental 
investigation of entropy rate measures, we were confronted with several challenges. First, how do we get reliable entropy rate estimates? This is crucial, since the calculation of entropy rate is reliable only for samples of sufficient sizes (Bentz 2017). Second, how do we compare entropy rate across age groups? Previous studies compared entropy rate (and similar measures) across languages using parallel texts, like the Bible, where the same content is translated into many languages. When the text is held constant, differences can more easily be attributed to structural differences between the languages and not to differences in content (Bentz et al., 2017, 2016; Ehret \& Szmrecsanyi, 2016; Juola, 2008; Koplenig et al., 2017; Lupyan, 2019; Lupyan \& Dale, 2010). However, we do not have such parallel texts at our disposal when doing developmental research (nor is it clear what such parallel texts would be for naturalistic interactions). Our third challenge was to ensure that differences in entropy rate between age groups will indeed reflect the effect of age, and not other factors. In what follows we outline our solutions in brief, and elaborate on each in subsequent sections. First, we used a stabilization criterion to determine the minimal sample size needed for reliable entropy rate estimates. In other words, we chose a sample size beyond which entropy rate remained almost unchanged. Each sample was composed of multiple conversations (a conversation is one unit of recording in the original corpora). In order to compensate for the fact that different age groups are not "parallel texts", we took multiple samples from each age group - the number of samples was also determined using a stabilization criterion. Finally, to make sure that any differences found between the samples from different ages indeed reflect age, rather than a sampling confound, we created an additional sampling procedure (the mixed-age condition), where we followed the same procedure but took samples from mixed ages: if entropy rate is impacted by the infants' age, then the change in entropy rate should disappear in this condition. The next sections describe these processes in detail.

Division into age groups. The NewmanRatner Corpus is already divided into different age groups, corresponding to different lab visits. In the Providence Corpus, however, children were recorded weekly/monthly. We could not measure entropy rate for each recording since it 
does not provide enough data for calculating reliable estimations of entropy rate (see next section) $)^{2}$. To overcome this, we grouped the recordings into two age bins: 12-24 months and 2436 months, resulting in two age points per child (The mean age across all transcripts and children is 18.25 months $(\mathrm{SD}=3.51)$ in the younger age group and 29.84 months $(\mathrm{SD}=3.32)$ in the older age group). The sampling procedures described below were applied separately to each age group in the NewmanRatner Corpus (3 groups in total), and to each age group for each child in the Providence Corpus (6 groups in total). After having established the age bins, we made them similar in size: if one age group had more conversations than another age group, we randomly selected a subset of the larger corpus, to equate it in size with the smaller one (see Tables 3 and 4 for total corpus size from which samples were taken for the different age groups).

Table 3: Number of words in each age group from which samples were taken in the NewmanRatner Corpus: each sample contained 10,000 words

\begin{tabular}{ccc}
\hline 7 months & 11 months & 24 months \\
\hline 92659 & 85794 & 90081 \\
\hline
\end{tabular}

Table 4: Number of words in each age group from which samples were taken in the Providence Corpus: each sample contained 20,000 words

\begin{tabular}{cccccc}
\hline \multicolumn{2}{c}{ Ethan } & \multicolumn{2}{c}{ Lily } & \multicolumn{2}{c}{ Naima } \\
\hline 1-2 years & $\mathbf{2 - 3}$ years & $\mathbf{1 - 2}$ years & $\mathbf{2 - 3}$ years & $\mathbf{1 - 2}$ years & $\mathbf{2 - 3}$ years \\
84991 & 79052 & 81456 & 80343 & 82726 & 81647 \\
\hline
\end{tabular}

Sample structure. We created each sample by randomly selecting conversations from each age group, and aggregating them in a random order. We only included utterances spoken by

${ }^{2}$ Looking at each conversation separately is not only too short in terms of data, but also from a theoretical perspective: it is unlikely that one conversation is a proper unit of analysis of IDS from the infant's perspective. 
caregivers. Importantly, since entropy rate is affected by the order words appear in, we kept each conversation intact 3 . The resulting sample was one long text of aggregated conversations (see Figure 1 for an illustration of the sampling procedure). Sample size was the same for all age groups (based on the stabilization criteria detailed in the next sections).

Determining sample size. As mentioned, the calculation of entropy rate is reliable only for samples of sufficient sizes (Bentz 2017). We followed the stabilization criterion used in Bentz et al., 2017 to determine sample size. In that paper, entropy rate was calculated for a gradually growing sample size. The sufficient sample size was defined as one where the SD of entropy rate in the next ten samples was smaller than or equal to 0.1, that is, where entropy rate did not change much with growing sample size. We applied the same procedure to our data. For each age bin, we gradually increased sample size, where each time the sample was 1000 words larger than the previous (1000, 2000, 3000...), until we reached the total number of words in that age bin. We then calculated entropy rate for each of these sample sizes, and calculated the SD of the entropy rate of each ten consecutive sample sizes, looking for the minimal corpus size (in words) where entropy rate did not change with further increase. This procedure was done for each age group separately. The final sample size was the one for which all age groups reached stabilization (e.g., if 10,000 words were needed for a reliable calculation at 7-months, but 20,000 were needed for 24-months, our selected sample size was 20,000 for all age bins). In practice, all age groups in both corpora required the same sample size, so no such adaptation was needed. The stabilization criterion procedure was implemented using the Hrate package (Bentz et al., 2017).

${ }^{3}$ Except for one conversation in each sample: Since the determined sample size was fixed for all samples and all age groups, the sample size was the cut-off point of the sample, even if it was in the middle of a conversation. For example, if the sample size determined for a corpus was 10,000 words, then each sample was of exactly 10,000 words. Because of this fixed word limit, each sample is very likely to have one conversation cut somewhere in the middle. Other than that, all conversations remained intact. 
Comparing entropy rate between age groups. To make sure samples are representative of the age group they are taken from, we took multiple samples from each age group4. This method was inspired by previous work that compared the complexity of non-parallel texts by taking multiple samples from each text (e.g., learner essays of beginners vs. advanced English learners, Ehret \& Szmrecsanyi, 2019). The number of samples was determined using a similar procedure as the one used to determine the minimal sample size. After finding the minimal reliable sample size (see previous section), we created 1000 samples of this size from the same age bin, increasing the number of samples by 10 each time (10 samples, 20 samples, 30 samples...). We then calculated the average entropy rate for each number of samples (e.g., average entropy rate for 10 samples: 5.57, average entropy rate for 20 samples: 5.6 , and so on). Finally, we calculated the SD of the average entropy rate of each five consecutive $\mathrm{N}$ of samples (10-50,60100,100-150...). The sufficient number of samples was defined as one where the SD of the average entropy rate in the next five samples was smaller than 0.01. For both corpora, the determined number of samples for each age bin was 100. We therefore randomly chose 100 samples out of the 1000 initial samples created for each age group. See supplementary material for the stabilization of entropy rates for both corpora.

\footnotetext{
${ }^{4}$ Instead of taking multiple samples from each age group, we could also treat all conversations within each age group as one big sample, such that we would only have one sample for each age group. Indeed, we repeated our sampling procedures with one big sample from each age group and the patterns of our results remain unchanged. This method is however problematic for two reasons: a. It doesn't allow for a statistical comparison between age groups, and b. Since entropy rate is affected by the order in which words appear, the way conversations are concatenated together can potentially impact the result. By taking multiple samples we control for that possible confound, since in each sample different conversations are grouped together in different order. This allows us to make sure that any age differences found cannot be driven by the particular order in which conversations were grouped together within each age group. We nevertheless conducted an additional analysis where each conversation appeared in only one sample. This method resulted in only a few samples per each age group (which is why we did not use it as our main analysis), but ensured that samples were maximally different from one another. We report the results of this alternative sampling procedure for each corpus below. Importantly, the results held in both sampling procedures.
} 


\section{Figure 1}

Illustration of the sampling procedures. Each dot represents IDS extracted from one conversation. Different colors represent different age groups $(X, Y, Z)$. Solid frames represent samples taken from different age groups: each frame is a random assembly of conversations taken from the same age group (the separate-age condition). The dashed frame represents a sample taken from the mixed-age condition: composed of randomly chosen conversations taken from different age groups (the mixed-age condition).

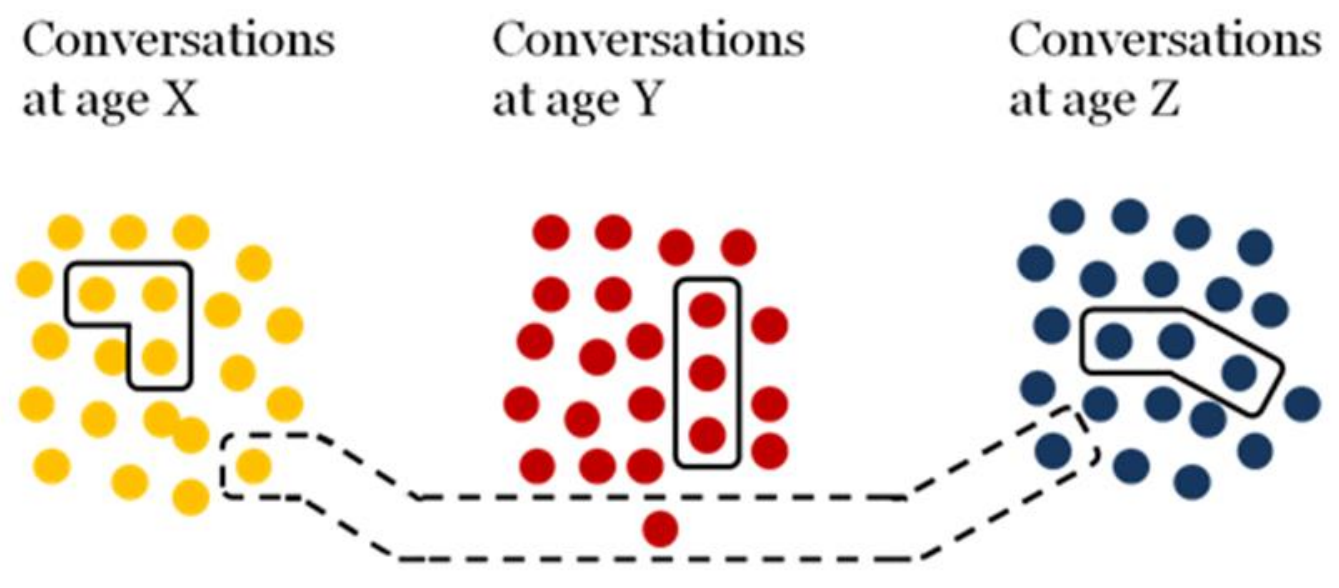

Variables of interest and hypothesis testing. After determining the sample size and the number of samples, we calculated entropy rate for each sample. To ensure that any difference in entropy rate stems from an effect of age, and is not simply the result of comparing multiple samples, we conducted another comparison where we aggregated conversations from different ages into the same sample. That is, we took the same number of samples (with the same sample size) but we allowed the mixing of conversations from different ages. We call this the mixed-age condition, in contrast with samples taken from separate ages - which we call the separate-age condition. The mixed-age condition had the same number of groups and the same number of samples as the separate-age condition. However, the groups in the mixed-age condition were not selected based on having the same age. In both conditions the procedure was the same: each sample was consisted of random sampling of conversations without replacement (that is, a conversation could not appear more than once in a given sample). If entropy rate is affected by 
the infant's age, then we should not see a developmental decrease in the mixed-age condition. See Figure 1 for an illustration of the sampling procedure in both conditions.

\section{Results}

Since we applied our sampling procedure separately to each corpus, and since each had a different division into age groups, we report the results for each corpus separately.

\section{The NewmanRatner Corpus}

Our stabilization criteria resulted in samples containing 10,000 words, and in taking 100 samples from each age group. We calculated entropy rate for each sample. As can be seen in Figure 2, entropy rate seems to increase with age: while entropy rate doesn't differ between 7-months (5.15) and 11-months (5.15), it is higher for 24 months (5.32). A one way ANOVA showed a significant effect of age $(\mathrm{F}(2)=116.7, p<0.001, \eta 2=0.44)$, indicating that, as predicted, parents speak less redundantly to older children. Simultaneous Tukey tests found the differences between 24 months and 7 months and between 24 months and 11 months to be significant, both at $p<0.001$ level. There was no difference, however, between 7 and 11 months $(p=0.99)^{5}$. Importantly, no change in entropy rate was found in our mixed-age condition $(5.29,5.29,5.28, \mathrm{~F}(2)=0.92, p=0.4$, $\eta 2=0.006)$, indicating that the differences in entropy rate reflect an effect of age, and are not a byproduct of comparing multiple samples.

\section{The Providence Corpus}

The Providence Corpus differs from the NewmanRatner Corpus, containing longitudinal naturalistic interactions between a small number of caregivers and children, engaged in various activities. Here, we investigate the entropy rate trajectory for each child separately. For this

${ }^{5}$ As mentioned in Footnote 4, we conducted an additional analysis where each conversation appeared in only one sample. This resulted in considerably fewer samples (8 per each age group). We calculated and compared entropy rate for each sample, and the pattern of results remain the same: no difference between 7 months (5.16) and 11 months (5.15), and an increase with age for 24 months (5.28). This was confirmed by a one way ANOVA $(\mathrm{F}(2)=6.58, \mathrm{p}=0.006, \mathrm{\eta} 2=0.38)$. 
corpus, the stabilization criteria resulted in samples of 20,000 words, and in taking 100 such samples from each group (recall there are six groups in this corpus: two age points for three different children). We calculated entropy rate for each sample. As can be seen in Figure 3, entropy rate increases with age for all three children (Ethan: 5.58, 5.89; Naima: 5.77, 5.89; Lily: $5.87,6.09)$. We ran a two-way ANOVA to compare the effect of child, age and their interaction on entropy rate. There was a significant effect of age $\left(F(1)=1007.4, p<0.001, \eta_{p}{ }^{2}=0.63\right)$, indicating that entropy rate increases with development; a significant effect child $(\mathrm{F}(2)=446.04, p<0.001$, $\left.\eta_{\mathrm{p}}{ }^{2}=0.6\right)$, indicating individual variation between children; and a significant interaction $(\mathrm{F}(2)=$ 66.96, $p<0.001, \eta_{\mathrm{p}}^{2}=0.18$ ), indicating individual variation in the developmental change in entropy rate ${ }^{6}$. In line with our prediction, entropy rate increased with age: speech directed to infants was less redundant as they were older. We next analyzed the results of the mixed-age condition. For the Providence corpus, the mixed-age condition was created by taking conversations from the same child, but from different ages. No differences in entropy rate were found between the mixed-age groups (Ethan: 5.86, 5.85; Lily: 5.86, 5.86; Naima: 5.85, 5.86; $\left.\mathrm{F}(1)=0.075, p=0.78, \eta_{\mathrm{p}}^{2}=0.0001\right)$, or between children $\left(\mathrm{F}(2)=1.8, p=0.16, \eta_{\mathrm{p}}^{2}=0.006\right)$. Again, finding no differences in the mixed-age condition indicates that the differences in entropy rate obtaining between age groups indeed stems from age (see Figure 3).

${ }^{6}$ Here also we conducted an additional analysis where each conversation appeared in only one sample. This resulted in 3 samples per each age of each child. We calculated entropy rate for each sample, and the pattern of results remained the same (Ethan: 5.54, 5.92; Naima: 5.78, 5.93; Lily: 5.9, 6.09). A two way ANOVA confirmed an effect of age $\left(\mathrm{F}(1)=40.53, p<0.001, \eta_{\mathrm{p}}{ }^{2}=0.77\right)$ and child $(\mathrm{F}(2)=16.26, p<0.001$, $\left.\eta_{\mathrm{p}}{ }^{2}=0.73\right)$. The interaction between them was marginally significant $\left(\mathrm{F}(2)=3.23, p=0.07, \eta_{\mathrm{p}}{ }^{2}=0.35\right)$. 


\section{Figure 2}

Entropy rate as a function of age in the NewmamRatner Corpus where (A) each group of samples are taken from a different age (separate-age condition) and (B) where age is shuffled between the groups (mixed-age condition). Each dot represents a sample, numbers represent group means.
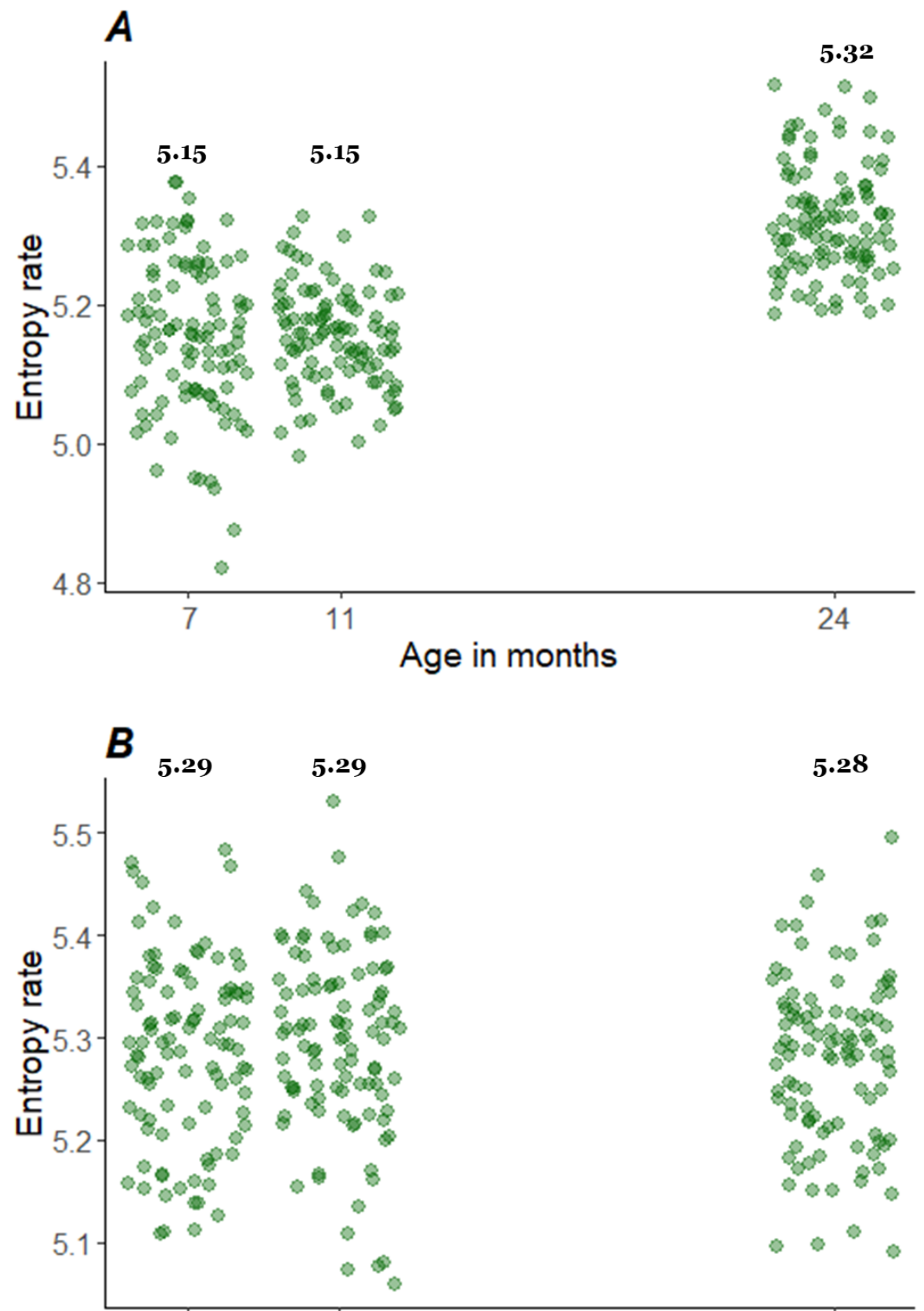

Shuffled age 


\section{Figure 3}

Entropy rate as a function of age and child in the Providence Corpus where (A) each group of samples within each child is taken from a different age (separate-age condition) and (B) where age is shuffled within each child (mixed-age condition). Each dot represents a sample, numbers represent group means.
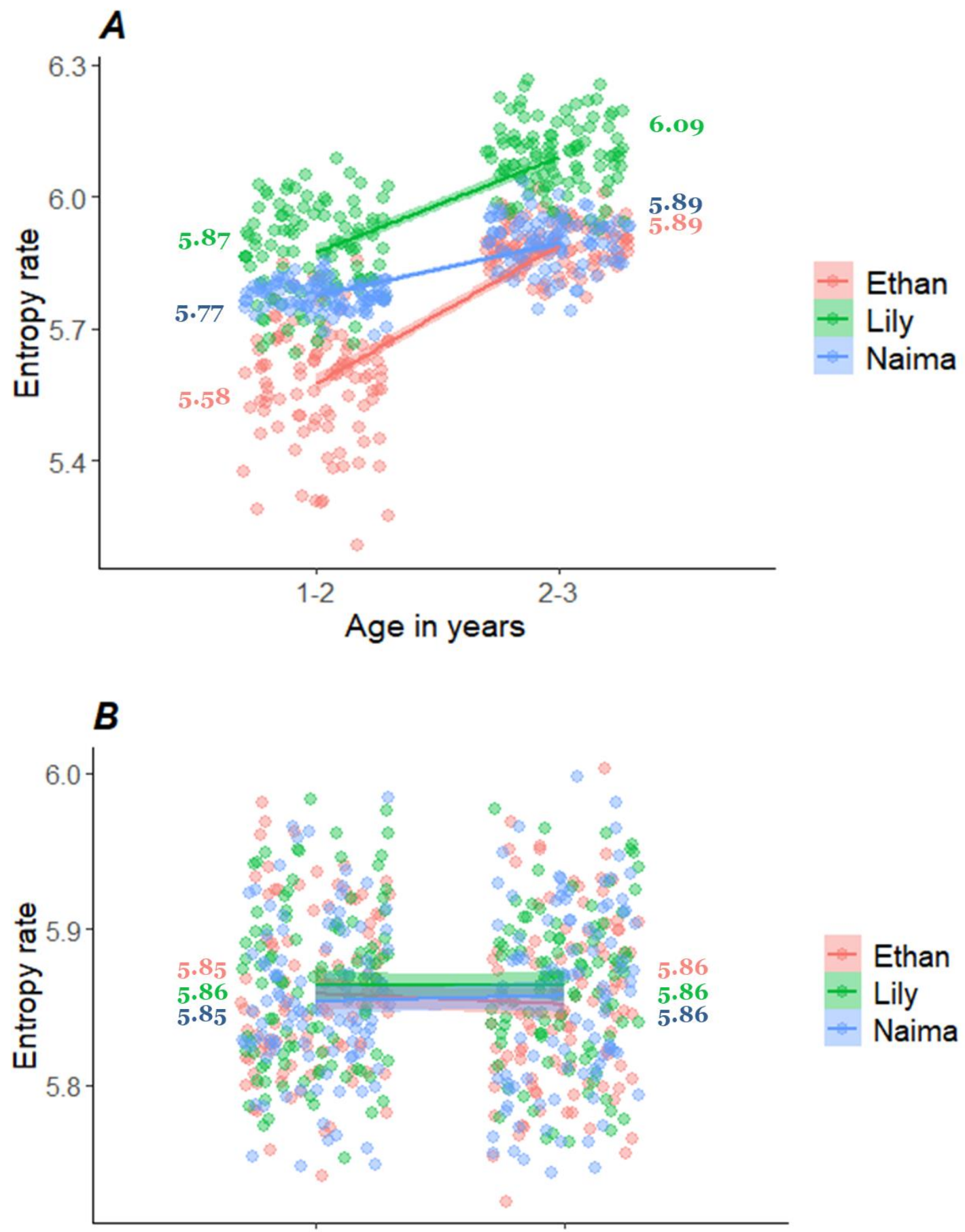

Ethan

Lily

Naima 


\section{Discussion}

In line with our prediction, we found an increase in entropy rate with age in both corpora, suggesting that parents use more redundant speech when talking to younger children across different conversational settings. These findings suggest that speakers are sensitive to the proficiency level of their interlocutors, and increase redundancy with less proficient interlocutors, in line with the communicative efficiency hypothesis (Buz et al., 2016; Jaeger \& Buz, 2017; Pate \& Goldwater, 2015). The findings also provide a new way to assess the overall level of redundancy in IDS: instead of investigating individual properties of IDS (lexical diversity, syntactic diversity), entropy rate offers a holistic evaluation of how repetitive - and therefore, predictable - is the input that young children hear.

But what drives the increased redundancy in younger ages? IDS has lower lexical diversity than in ADS, with lexical diversity increasing with age (Schwab \& Lew-Williams, 2016a; Soderstrom, 2007). The decrease in redundancy we found could reflect the increase in lexical diversity that occurs with development. However, IDS has another property that could be driving the increase in entropy rate: IDS has substantial repetition of multi-word sequences, in the form of frequent frames or repeated chunks (Arnon, 2016; Cameron-Faulkner et al., 2003; Ferrier, 1978; Stoll et al., 2009). These larger sequences are claimed to play an important role in language learning by providing preferred patterns for multi-word production (Bannard \& Matthews, 2008; Lieven et al., 2003, 2009), and serving as early building blocks for learning morphological and syntactic regularities (Abbot-Smith \& Tomasello, 2006; Arnon et al., 2017; Arnon \& Clark, 2011; Reali \& Christiansen, 2007; Skarabela et al., 2021). Such units are predicted to be relied on less in later development and in second language learning (Arnon \& Christiansen, 2017; Siegelman \& Arnon, 2015). Importantly, both types of repetitions (lexical and multi-word) will make the input more redundant and will be reflected in a lower entropy rate. Recall the comparison of the three texts in Section 1.1: text [A] has a lower entropy rate than text [B] because it has more repetitions of words and multi-word sequences, while text [C] has a higher entropy rate than text [B] only 
because of multi-word sequences: both texts have the same lexical diversity, but text [B] consists of two multi-word chunks while text [C] does not.

In Study 2 we investigate which properties underlies the differences in redundancy we found. Specifically, we examine the role of multi-word sequences in these differences. We do this by creating an additional comparison condition where in each sample words are shuffled within each sentence. If the increase in entropy rate is driven only by an increase in lexical diversity, then the effect should not be impacted by ruining the order in which words appear in sentences. If, however, input to younger children is more redundant also due to having more repetitions of multi-word sequences, then the difference between age groups should become smaller (or disappear) once words within sentences are shuffled (since this shuffling will ruin the repetition of multi-word sequences).

\section{Study 2}

Study 2 set out to assess the impact of lexical vs. multi-word repetition on the increase in entropy rate found in Study 1. We can contrast three different predictions. First, since lexical diversity (measured by type-token ratio) increases with age (Schwab \& Lew-Williams, 2016a; Soderstrom, 2007), the effect we found in Study 1 could reflect parents' use of a more diverse vocabulary with their children as they grow older. If this is the only effect driving the results in Study 1, then shuffling words within each sentence should not impact the entropy-rate effect: diversity of lexicon is not impacted by the order in which words appear in a sentence. A second possibility is that the results of Study 1 are driven by a developmental decrease in repetitions of multi-word sequences, rather than a decrease in lexical repetitions. If the results of Study 1 are driven only by multi-word repetitions, then an increase in entropy rate should not be found when the words in each sentence are shuffled. Finally, if the results of Study 1 are driven by both types of repetitions, then ruining the order in which words appear in sentences should still result in an increase in entropy rate, but entropy rate measures should be overall higher compared to the non- 
shuffled text, since one of the factors contributing to redundancy in the input (repetitions of multiword sequences) will no longer be there.

\section{Method}

\section{The corpora}

We used the same corpora as in Study 1.

\section{Procedure}

We used the same sampling procedure as in Study 1 (taking 100 samples from each age bin in each corpus), but now in each sample we shuffled the words within each sentence before calculating entropy rate7. That is, within each sample (taken from multiple conversations within each age group; 10,000 words in the NewmanRatner Corpus and 20,000 words in the Providence Corpus) we shuffled the words in each sentence (but sentences still occurred in the same order as in the original conversation they belonged to). Importantly, the samples were taken anew (that is, the samples taken in Study 2 were not identical to the ones taken in Study 1). The shuffling procedure was conducted using the sample() function in $\mathrm{R}$ ( $\mathrm{R}$ Core Team, 2019). By shuffling words in this way, we removed their sequential position in a sentence, but left their lexical diversity intact.

\section{Results}

\section{The NewmanRatner Corpus}

Figure 4 shows entropy rate across age bins when words were shuffled within each sample. As can be seen, while there seems to be differences in entropy rate between the three ages, these differences are smaller when compared to Study 1 (7 months: 5.76, 11 months: 5.69, 24 months: 5.81). In addition, entropy rates are higher overall compared to those found in Study 1: by ruining the order in which words appeared in sentences in the original conversations, the texts were now

\footnotetext{
7 We used the corpora's existing segmentation to sentences.
} 
less predictable and therefore less redundant. A one-way ANOVA revealed an effect of age $(\mathrm{F}(2)=$ $72.61, p<0.001, \eta 2=0.2)$. Simultaneous Tukey tests found the differences between 24 months and 7 months and between 24 months and 11 months to be significant, both at $\mathrm{p}<0.001$ level. There was no difference, however, between 7 and 11 months $(\mathrm{p}=0.44)$. Note that although the difference between 7 and 11 months is not significant, there seems to be a trend of a decrease in entropy rate between these ages. We believe this pattern reflects an idiosyncratic property of this corpus: the fact that lexical diversity in the corpus as a whole (measured by type-token ratio) does not increase with age (see Table 1), in contrast with the more common developmental pattern (Soderstrom, 2007). The lack of a developmental increase in lexical diversity in this corpus is most likely due to its specific characteristics: in this corpus, mothers and children of different ages were invited to the lab to play with the same box of toys. This setup most likely confines lexical diversity, thereby placing an upper bound on its increase.

We directly compare the impact of age on entropy rate in Study 1 and Study 2, by running a two-way ANOVA on a pooled dataset of both samples. Samples were coded as "non-shuffled" (Study 1) or "shuffled" (Study 2). A two-way ANOVA with study (non-shuffled vs. shuffled), age and their interaction revealed a significant effect of age $\left(F(2)=200.3, p<0.001, \eta_{p}^{2}=0.4\right)$, indicating that entropy rate is impacted by age. There was also a significant effect of study $(\mathrm{F}(1)=$ $7734.5, p<0.001, \eta_{\mathrm{p}}{ }^{2}=0.93$ ), showing that entropy rate was higher in Study 2 (where words in each sample were shuffled within each sentence), indicating the samples were less predictable. There was also a significant interaction between age and study, $\left(\mathrm{F}(2)=28.65, p<0.001, \eta_{\mathrm{p}}{ }^{2}=0.09\right)$, indicating that the impact of age on entropy rate is stronger in Study 1.

In sum, the findings from the NewmanRatner Corpus show that entropy rate increase with age when the words within sentences are shuffled, but the effect of age is smaller than in Study 1. This suggests the decrease in redundancy is not only affected by an increase in lexical diversity, but also by a decrease in repetitions of multi-word sequences. 


\section{Figure 4}

Entropy rate as a function of age in the NewmanRatner Corpus when each sentence in each sample is shuffled for words (Study 2) compared to when no such shuffling occurs (Study 1). Each dot represents a sample, numbers represent group means.

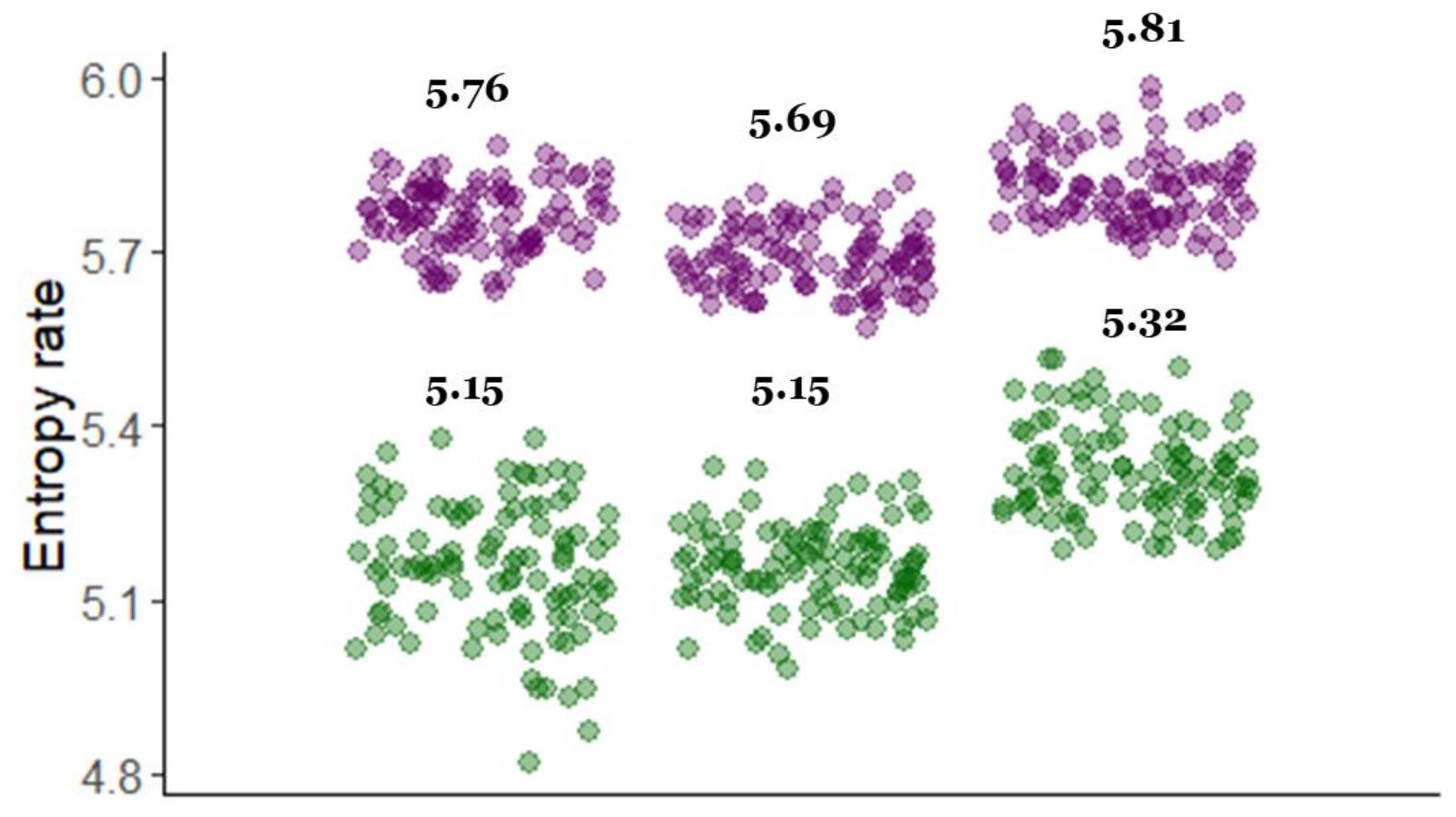

\section{Age in months}

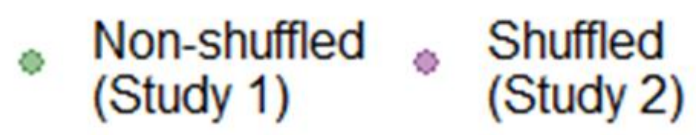

Overall, the fact that the NewmanRatner corpus shows no decrease in lexical repetitiveness (in contrast to the more typical state of affairs), contributes an interesting and important angle to the current findings: we find an increase in entropy rate despite the fact that this corpus does not show the typical decrease of lexical repetitiveness. This highlights even further the decrease of redundancy over age, and in particular the role of multi-word repetitiveness in driving this decrease. Taken together, these results point to the role of multiword units in the developmental decrease in redundancy in IDS. 


\section{The Providence Corpus}

Figure 5 shows entropy rate after word-shuffling for the Providence corpus. As in the NewmanRatner corpus, we still find here an increase of entropy rate with age, albeit the increase seems to be slightly smaller (Ethan: 6.15,6.42; Lily: 6.42,6.58; Naima: 6.33,6.42). Entropy rates were higher overall compared to the ones found in Study 1, indicating that the input becomes less predictable (and therefore, less redundant) as words are divorced from their original sequential position in the sentence. A two-way ANOVA with child, age and their interaction as factors confirmed there was a significant effect of age $\left(\mathrm{F}(1)=1014.6, p<0.001, \eta_{\mathrm{p}}{ }^{2}=0.63\right)$, indicating a developmental increase in entropy rate; a significant effect of child $\left(F(2)=516.3, p<0.001, \eta_{p}{ }^{2}\right.$ $=0.63$, indicating individual variation in entropy rate; and their interaction $(\mathrm{F}(2)=103.2$, $p<0.001, \eta_{\mathrm{p}}^{2}=0.26$ ), indicating a different effect of age on entropy rate for the different children. To directly compare Study 1 and Study 2, we ran an ANOVA on the pooled dataset, coding each sample for the study it came from (non-shuffled (Study 1) or shuffled (Study 2)). We used a threeway ANOVA to compare the effect of study (non-shuffled vs. shuffled), age, child and their interactions on entropy rate. There was a significant effect of age $\left(F(1)=1997, p<0.001, \eta_{p}^{2}=0.63\right)$, indicating a developmental increase in entropy rate across studies; a significant effect of child $\left.\mathrm{F}(2)=941.85, p<0.001, \eta_{\mathrm{p}}{ }^{2}=0.61\right)$, indicating individual variation; and a significant effect of study $\left(F(1)=15049.2, p<0.001, \eta_{p}^{2}=0.93\right.$ ), indicating higher entropy rates for Study 2 (as, again, shuffling the samples render the input less redundant). Finally we found all the interactions were significant: age and child: $\mathrm{F}(2)=161.14, p<0.001, \eta_{\mathrm{p}}{ }^{2}=0.21$, indicating a different age effect for different children; age and study: $\mathrm{F}(1)=23.43, p<0.001, \eta_{\mathrm{p}}^{2}=0.02-$ indicating that the effect of age is larger in Study 1 compared to Study 2 (though note the small effect size); and child and study: $\mathrm{F}(2)=5.2, p=0.006, \eta_{\mathrm{p}}{ }^{2}=0.008$, indicating children had different entropy rates in the different studies. 


\section{Figure 5}

Entropy rate as a function of age and child in the Providence Corpus when each sentence in each sample is shuffled for words (Study 2, darker shades) compared to when no such shuffling occurs (Study 1, lighter shades). Each dot represents a sample, numbers represent group means.

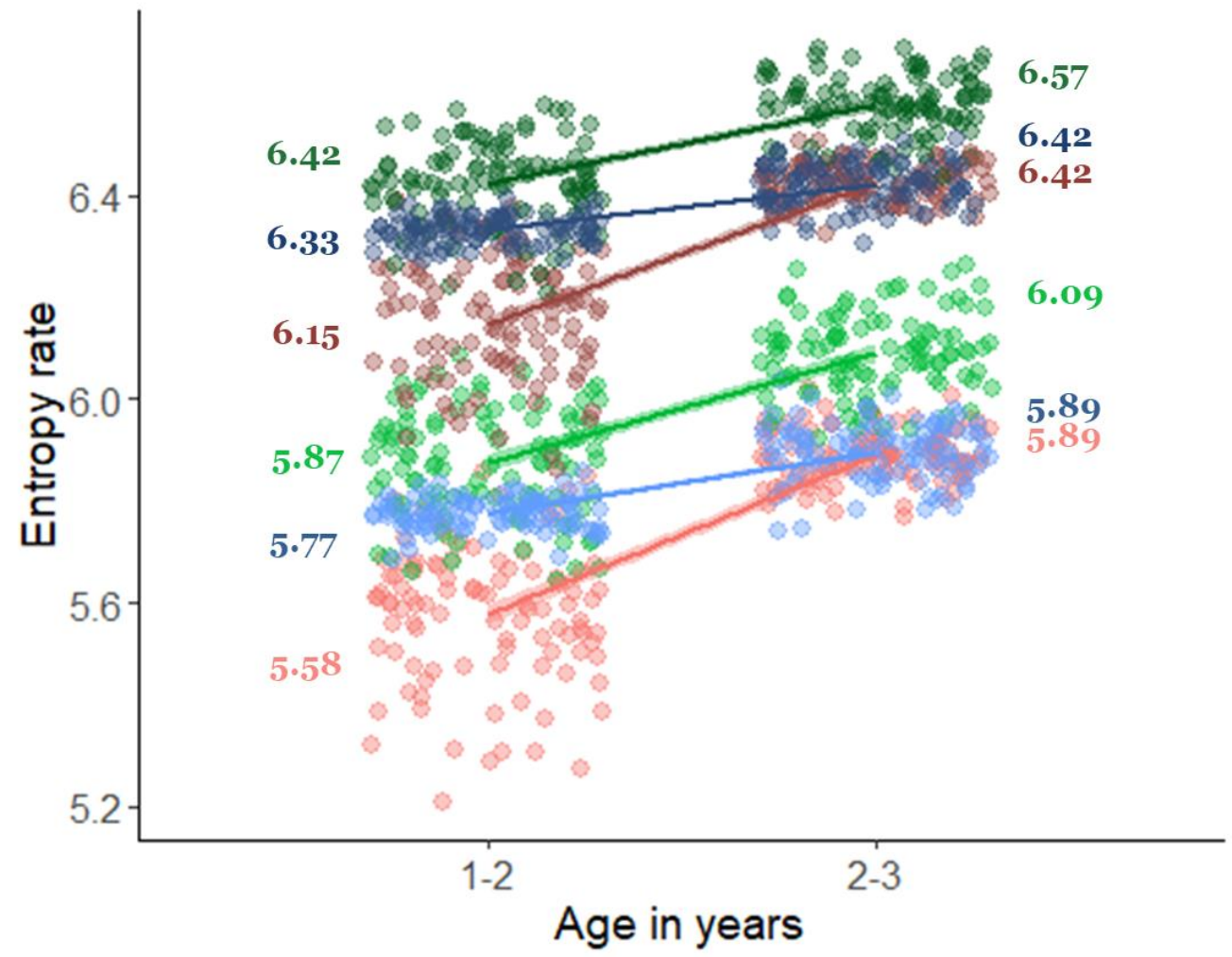

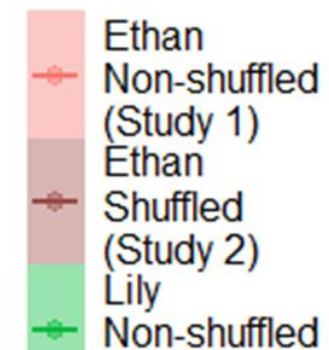

(Study 1)

Lily

Shuffled

(Study 2)

Naima

Non-shuffled

(Study 1)

Naima

Shuffled

(Study 2)

In sum, for two of the three children, entropy rate still increased with age when the words in the samples were shuffled, but the effect of age was smaller than in Study 1. In other words, the increase in redundancy seems to be affected both by an increase in lexical diversity and a decrease in repetitions of multi-word sequences.

\section{Discussion}

Study 2 set out to investigate what underlies the decrease in redundancy found in both corpora in Study 1. By shuffling words within each sample, we removed the possible contribution of multiword repetitiveness, allowing us to tease apart the effect of lexical repetitiveness and multi-word repetitiveness (both characteristic of IDS; Cameron-Faulkner et al., 2003; Schwab \& Lew- 
Williams, 2016; Soderstrom, 2007). Our results suggest that the developmental increase in entropy rate found in Study 1 reflects not only an increase in lexical diversity, but also a decrease in repetitions of multi-word sequences. In both the NewmanRatner Corpus and the Providence Corpus, there was still an increase in entropy rate, but a smaller one: suggesting that lexical repetitiveness drives some of the effect in Study 1, but not all of it. That is, repetitions of multiword sequences contribute to the developmental effect found in Study 1 for both corpora.

\section{General Discussion}

Speakers generally tend to avoid redundancy during language use when it is possible (Jaeger, 2010; Levy \& Jaeger, 2007; Mahowald et al., 2013; Piantadosi et al., 2011b; Tily \& Piantadosi, 2009). However, according to the communicative efficiency hypothesis, speakers are predicted to increase redundancy with increased comprehension difficulty. Speakers should therefore speak more redundantly when their interlocutors can potentially misunderstand them. Importantly, speakers are known to modify their speech on the basis of global estimations of their interlocutors (Arnold, 2008; Loy \& Smith, 2020). However, very few studies have investigated whether speakers adapt the redundancy in their speech based on such global properties. Our study set out to ask whether speakers speak more redundantly with interlocutors that are perceived to have difficulty in understanding, specifically, infants learning their first language. We examined redundancy developmentally in IDS, with two goals in mind: (1) Providing a novel measure of global redundancy in IDS, and (2) Testing whether redundancy is impacted by the interlocutors' perceived overall proficiency level. We used an information-theoretic measure called entropy rate, which was previously used to compare language complexity cross-linguistically (Bentz et al., 2017), but has not been used to compare different types of speech within a language. This measure provided us with a global estimate of redundancy that is sensitive to the order of words: texts where words are more predictable given previous words will have a lower entropy rate, reflecting greater redundancy. 
Applying this measure to samples of IDS taken from infants of different ages, we found that, in line with our predictions, parents tend to speak more redundantly to their children when they are younger. These results are compatible with recent findings in the phonetic domain, showing that adult speakers provide more redundant acoustic signals when talking to infants compared to proficient adult speakers (Pate \& Goldwater, 2015; Tippenhauer et al., 2020; Uther et al., 2007). Our results expand these findings in two important ways. First, they broaden the scope of speakers' adaptations from the phonetic level to the word and multi-word level. Second, they show that differences in redundancy are not only found when talking to infants as opposed to adults (a salient distinction that is likely to be computationally easy for the speaker), but also when talking to children of different ages. They illustrate speakers' fine-grained sensitivity to the proficiency level of their interlocutors. These results are compatible with other findings showing that adult modifications in IDS are impacted by infants' age (for example, re-using children's words more the younger they are, Snow, 1972; Yurovsky, Doyle, \& Frank, 2016).

In Study 2 we set out to test which properties of IDS drive the developmental decrease in redundancy. IDS is characterized both by lower lexical diversity (Soderstrom, 2007), and by having frequently recurring multi-word sequences (Arnon, 2016; Cameron-Faulkner et al., 2003; Stoll et al., 2009). While previous studies document a developmental decrease in lexical repetitiveness (Schwab \& Lew-Williams, 2016a; Soderstrom, 2007), there is no parallel evidence for a decrease in repetitions of multi-word units. By measuring entropy rate on samples where words were shuffled, we could tease apart the effect of lexical and multi-word repetitiveness on entropy rate. We found that in both tested corpora, the developmental decrease in redundancy was not a mere reflection of an increase in lexical diversity. Rather, the decrease in redundancy was largely impacted by a decrease in repetitions of multi-word sequences. This finding serves as the first indication that repetitions of multi-word sequences decrease with infant's age, and moreover, that this decrease makes the input globally less redundant. In what follows, we discuss the relevance of our findings for communicative efficiency and language development. 


\section{Communicative efficiency}

According to the communicative efficiency hypothesis, speakers should speak more redundantly when there is increased comprehension difficulty. This may happen when the message is unpredictable in context (Aylett \& Turk, 2004; Kurumada \& Jaeger, 2015; Levy \& Jaeger, 2007; Mahowald et al., 2013); when there is high environmental noise (Summers et al., 1988; Zhao \& Jurafsky, 2009); or when the interlocutor has difficulty decoding the message (Buz et al., 2016). Several studies demonstrate the impact of the interlocutor's perceived difficulty in local cases of misunderstanding within a conversation (Buz et al., 2016; Lockridge \& Brennan, 2002; Roche et al., 2013). However, only a handful of studies show an increase in redundancy dependent on global characteristics of interlocutors, and all of them focused on the phonetic domain (Pate \& Goldwater, 2015; Tippenhauer et al., 2020; Uther et al., 2007). Our results indicate that the tendency to reduce or increase redundancy is impacted by the interlocutor, and that this impacts speech beyond articulation. Specifically, speakers seem to increase redundancy when conversing with younger language learners. This finding opens up new questions about how perceived proficiency impacts different kinds of linguistic redundancy, and whether changes in redundancy are applied similarly to different kinds of learners.

With regards to the type of redundancy, we found a developmental decrease in redundancy in the word and multi-word level, with stronger effects from the multi-word level. Other domains, such as morphosyntax, where communicative efficiency has been shown to impact production choices, could also be modified when talking to younger learners. For instance, we might see fewer morphological reductions (e.g, isn't vs. is not, Frank \& Jaeger, 2008) or reduced omission of optional syntactic words (e.g., optional case marking, Kurumada \& Jaeger, 2015) in speech directed to younger children compared to speech directed to older children or adults. We are currently conducting several corpus-studies to test the impact of the interlocutor on redundancy in morphosyntactic domains. 
A second open question has to do with how proficiency is perceived and how interlocutor type impacts changes in redundancy. In the current study, we tested the impact of conversing with young language learners on redundancy, where their lower proficiency is deduced from their age. Additional properties of interlocutors could also impact the trade-off between minimizing effort and maximizing understandability. Within a developmental setting, other factors beyond age could indicate lower proficiency: for example, speech directed to late talkers could be more redundant than speech directed to more proficient talkers of the same age. Between adults, conversations between friends are typically based on more common knowledge compared to conversations between strangers (Fussell \& Krauss, 1989), which could make them less redundant. More broadly, if increased redundancy is driven by the perceived proficiency level of the interlocutor, rather than interlocutors' age, then we should find similar changes in redundancy in speech directed to adult learners. The literature provides two contrasting predictions for whether we modify redundancy similarly in speaking with children and adult L2 learners. On the one hand, we may not produce more redundant speech when conversing with adult L2 learners: adult learners have cognitive capacities similar to those of adult native speakers, and might therefore be perceived as more competent, despite their low proficiency level in the language in question. In addition, a recent hypothesis predicts such differences on the basis of the idea that children and adults might have different learning constraints: the Linguistic Niche Hypothesis suggests that child learners benefit from redundancy, but adult learners do not, or at least not to the same degree (Dale \& Lupyan, 2012; Lupyan, 2019; Lupyan \& Dale, 2010). Following this hypothesis, speech directed to child learners is predicted to be more redundant than speech direct to adult learners, because of speakers' adaptation to these different learning constraints. On the other hand, the impact of the interlocutor on redundancy might be strictly related to perceived language proficiency, regardless of age. If this is the case, then the differences found between age groups in the current study should be replicated with adult learners. Specifically, speech directed to adult learners should be more redundant compared to speech directed to adult proficient 
speakers. However, it is not easy to find suitable corpora to test this comparison since the calculation of entropy rate requires samples of sufficiently large sizes (Bentz et al., 2017), of which there are very few for L2-directed speech. Moreover, the comparison requires parallel corpora of speech directed to adult learners vs. proficient speakers (for example, two sets of corpora that contain similar conversational content, but differ in the proficiency level of the interlocutors). However, a separate study we conduct on a different sort of redundancy supports the prediction above. In this study we compare participants' descriptions of the same picture book to child learners of different ages, adult learners and adult proficient speakers. We find that speakers use more redundant references (e.g., the boy rather than he) when their interlocutors are learners compared to proficient speakers. Importantly, the same pattern is found for both child and adult learners, illustrating the effect is not (only) driven by general cognitive maturation processes of the listeners, but rather by language proficiency (Tal et al., 2023).

\section{Language development}

The language acquisition literature highlights the prevalence and the facilitative role of different types of repetitions in IDS: lexical repetition, frequently recurring phrases, variation sets and repeated chunks (Arnon, 2016; Cameron-Faulkner et al., 2003; Küntay \& Slobin, 1996; Schwab \& Lew-Williams, 2016a; Stoll et al., 2009). Importantly, these properties are typically investigated separately. Here, we provided a novel measure to assess the overall redundancy in IDS, and to investigate its developmental trajectory. Having shown that IDS becomes less redundant with development, we found that this effect is largely driven by repetitions of multi-word units. This finding joins the growing literature on the prevalence and importance of multi-word sequences in language development (Abbot-Smith \& Tomasello, 2006; Arnon, 2016, 2021; Arnon \& Christiansen, 2017; Stoll et al., 2009). There is growing evidence that multi-word sequences are building blocks for language learning (Arnon \& Clark, 2011; Bannard \& Matthews, 2008; Fernald \& Hurtado, 2006; Skarabela et al., 2021). Children and adult learners are claimed to differ in their reliance on multi-word sequences in learning, a tendency that can explain some of the difference 
between L1 and L2 learning (Arnon, 2021; Arnon \& Christiansen, 2017; Arnon \& Ramscar, 2012; Siegelman \& Arnon, 2015). Consequently, multi-word sequences are predicted to be more common in the inventory of L1 learners compared to adult L2 learners (Arnon \& Christiansen, 2017; McCauley \& Christiansen, 2014). This prediction is supported by the current study: we show here for the first time that repetitions of multi-word units in IDS decrease as the child grows older. Taken together, this finding provides additional evidence for the role of multi-word sequences in IDS. The higher frequency of multi-word repetitiveness at younger ages might aid young children's reliance on such constructions in language learning (Goldberg, 2006; Tomasello, 2003). Investigating the contribution of multi-word sequences to the increased redundancy in children's input provides a new way to test theoretical predictions regarding their prominence in language acquisition.

Finally, the current study provides an important first step in examining redundancy developmentally in IDS using an information theoretic framework. As such, it has several limitations and raises additional questions to pursue in future work. First, our oldest infants were 36 months old. At some point, speech directed to children should reach similar redundancy rates to speech directed to adults. When are children perceived as proficient enough for adult-like redundancy? To get an initial insight into this question, we can look at a previous study that calculated entropy rate for adult language: Bentz et al. (2017) looked at translations of the same high-register written texts in various languages, and found that entropy rates across languages display relatively narrow distributions. English (the language we looked at here) seems to be close to the mean value: 5.97 . Interestingly. this value is very close to what we found for the 24-36 months in the Providence Corpus (5.89-6.09), so it could be tempting to suggest that by the age of three children are starting to be perceived as adult-like in terms of their comprehension. However, we are hesitant to draw such conclusions (even preliminary or impressionistic ones), due to the large differences between the type of texts looked at in the current study (spontaneous spoken conversations between caregivers and infants), and the ones used by Bentz et al. 2017 (e.g., 
the Bible). A comprehensive comparison of entropy rate between IDS and adult speech should involve texts and contexts that are as similar as possible. Otherwise, it would be difficult to assess whether differences found are attributed to the different interlocutors or some other property of the conversation (Ehret \& Szmrecsanyi, 2016, 2019; Juola, 2008).

A second important way to broaden the current findings is to look at the developmental trajectory of entropy rate in languages other than English. Using a global measure of redundancy such as entropy rate provides as a novel way to make cross-linguistic comparisons and form novel predictions. For example, it would be instructive to look at morphologically complex languages, where, compared to English, more information is expressed within words rather than using multiword constructions. While we should still expect to see an overall decrease in entropy rate with development, we predict to find a different trade-off between lexical and multi-word repetitiveness driving this decrease. We are currently investigating these predictions.

\section{Conclusion}

According to the communicative efficiency hypothesis, speakers aim to balance two competing pressures: minimizing production effort and maximizing understandability. While typically avoiding redundancy, speakers produce 'costlier' signals when understandability is at risk. Here, we asked whether conversing with learners (interlocutors who are typically perceived to have difficulty in comprehension) results in more redundant language. We tested this by quantifying redundancy in infant-directed speech using entropy rate - an information-theoretic measure reflecting the average redundancy of texts. We found that, as predicted by principles of efficient communication, speakers use more redundancy the younger their infant addressees are. This increased redundancy in younger ages was found to be largely driven by repetitions of multiwords sequences. This serves as a first indication for a decrease in such sequences over age, and joins a great volume of literature pointing at the prominent role of multi-word sequences in language learning. 


\section{Supplementary material}

Supplementary data to this paper and the analysis code used to generate all reported results are available here:

\section{https://osf.io/kj6mg/?view only=97ofccgfc4fo4940a2821b74e031e51c}

\section{Acknowledgments}

We would like to thank Christian Bentz, Henry Brice, Haim Dubossarsky and Noam Siegelman for helpful discussions. We thank Noam Siegelman and Haim Dubossarsky for feedback on a previous version of this paper. This research was supported by the Israeli Science Foundation grant number 584/16 awarded to IA. ST was funded by the Mandel Scholion Center at the Hebrew University of Jerusalem.

\section{References}

Abbot-Smith, K., \& Tomasello, M. (2006). Exemplar-learning and schematization in a usagebased account of syntactic acquisition. Linguistic Review, 23(3), 275-290. https://doi.org/10.1515/TLR.2006.0011

Ariel, M. (1990). Accessing noun-phrase antecedents. Routledge.

Arnold, J. E. (2008). Reference production: Production-internal and addressee-oriented processes. Language and Cognitive Processes, 23, 495-527. https://doi.org/10.1080/01690960801920099

Arnon, I. (2016). The nature of child-directed speech in Hebrew: frequent frames in a morphologically rich language. In R. A. Berman (Ed.), The Acquisition of Hebrew (pp. 201-223). John Benjamins.

Arnon, I. (2021). The Starting Big approach to language learning. Journal of Child Language, 122. https://doi.org/10.1017/So305000921000386

Arnon, I., \& Christiansen, M. H. (2017). The role of multiword building blocks in explaining L1L2 differences. Topics in Cognitive Science, 9(3), 621-636. 
https://doi.org/10.1111/tops.12271

Arnon, I., \& Clark, E. V. (2011). Why brush your teeth is better than teeth - children's word production is facilitated in familiar sentence-frames. Language Learning and Development, 7 (2), 107-129. https://doi.org/10.1080/15475441.2010.505489

Arnon, I., McCauley, S. M., \& Christiansen, M. H. (2017). Digging up the building blocks of language: Age-of-acquisition effects for multiword phrases. Journal of Memory and Language, 92, 265-280. https://doi.org/10.1016/j.jml.2016.07.004

Arnon, I., \& Ramscar, M. (2012). Granularity and the acquisition of grammatical gender: How order-of-acquisition affects what gets learned. Cognition, 122(3), 292-305. https://doi.org/10.1016/j.cognition.2011.10.009

Aylett, M., \& Turk, A. (2004). The Smooth Signal Hypothesis: A functional explanation for relationships between redundancy, prosodic prominence, and duration in spontaneous speech. Language and Speech, 47(1), 31-56.

Bannard, C., \& Matthews, D. (2008). Stored word sequences in language learning of four-word combinations. Psychological Science, 19(3), 241-248.

Bentz, C., Alikaniotis, D., Cysouw, M., \& Ferrer-i-Cancho, R. (2017). The entropy of wordsLearnability and expressivity across more than 1000 languages. Entropy, 19(6), 1-32. https://doi.org/10.3390/e19060275

Bentz, C., Ruzsics, T., Koplenig, A., \& Samardži, T. (2016). A comparison between morphological complexity measures: typological data vs. language corpora. Proceedings Ofthe Workshop on Computational Linguistics for Linguistic Complexity, 142-153. https://www.aclweb.org/anthology/papers/W/W16/W16-4117/

Brennan, S. E., \& Hanna, E. (2009). Partner-specific adaptation in dialog. Topics in Cognitive Science, 1, 274-291. https://doi.org/10.1111/j.1756-8765.2009.01019.x

Bresnan, J., Cuetni, A., Nikitiana, T., \& Baayen, R. H. (2007). Predicting the Dative Alternation. In G. Boume, I. Kraemer, \& J. Zwarts (Eds.), Cognitive foundations of interpretation (pp. 
69-94). Royal Netherlands Academy of Science.

Buz, E., Tanenhaus, M. K., \& Jaeger, T. F. (2016). Dynamically adapted context-specific hyperarticulation: Feedback from interlocutors affects speakers 'subsequent pronunciations. Journal of Memory and Language, 89, 68-86. https://doi.org/10.1016/j.jml.2015.12.009

Cameron-Faulkner, T., Lieven, E., \& Tomasello, M. (2003). A construction based analysis of child directed speech. Cognitive Science, 27(6), 843-873.

https://doi.org/10.1016/j.cogsci.2003.06.001

Chafe, W. L. (1994). Discourse, consciousness, and time. Chicago University Press.

Clark, H. H., \& Murphy, G. L. (1983). Audience design in meaning and reference. In J. F. LeNy \& W. Kintsch (Eds.), Language and Comprehension (pp. 287-299). North-Holland Publishing Co.

Cohen Priva, U. (2015). Informativity affects consonant duration and deletion rates. Laboratory Phonology, 6(2), 243-278. https://doi.org/10.1515/lp-2015-00o8

Dale, R., \& Lupyan, G. (2012). Understanding the origins of morphological diversity: the linguistic niche hypothesis. Advances in Complex Systems, 15(3), 1150017-1-1150017-16.

Demuth, K., Culbertson, J., \& Alter, J. (2006). Word-minimality, epenthesis, and coda licensing in the acquisition of English. Language and Speech, 49(2), 137-174.

Ehret, K. (2018). An information-theoretic view on language complexity and register variation: Compressing naturalistic corpus data. Corpus Linguistics and Linguistic Theory. https://doi.org/10.1515/cllt-2018-0033

Ehret, K., \& Szmrecsanyi, B. (2016). An information-theoretic approach to assess linguistic complexity. In R. Baechler \& G. Seiler (Eds.), Complexity, Isolation, and Variation (pp. 7194). de Gruyter.

Ehret, K., \& Szmrecsanyi, B. (2019). Compressing learner language: An information-theoretic measure of complexity in SLA production data. Second Language Research, 35(1), 23-45. https://doi.org/10.1177/0267658316669559 
Ferdinand, V., Kirby, S., \& Smith, K. (2019). The cognitive roots of regularization in language. Cognition, 184, 53-68. https://doi.org/10.1016/j.cognition.2018.12.002

Fernald, A., \& Hurtado, N. (2006). Names in frames: infants interpret words in sentence frames faster than words in isolation. Developmental Science, 9(3), 33-40.

Ferreira, V. S. (2008). Ambiguity, accessibility, and a division of labor for communicative success. Psychology of Learning and Motivation, 49, 209-246. https://doi.org/10.1016/Soo79-7421(08)00006-6

Ferrier, L. J. (1978). Some observations of error in context. In N. Waterson \& C. Snow (Eds.), The development of communication (pp. 301-309). Wiley.

Frank, A. F., \& Jaeger, T. F. (2008). Speaking Rationally : Uniform Information Density as an Optimal Strategy for Language Production. The 3oth Annual Meeting of the Cognitive Science Society, 939-944.

Fussell, S. R., \& Krauss, R. M. (1989). Understanding friends and strangers: the effects of audience design on message comprehension. European Journal of Social Psychology, 19(6), 509-525. https://doi.org/10.1002/ejsp.2420190603

Gibson, E., Futrell, R., Piandadosi, S. T., Dautriche, I., Mahowald, K., Bergen, L., \& Levy, R. (2019). How Efficiency Shapes Human Language. Trends in Cognitive Sciences, 23(5), 389-407. https://doi.org/10.1016/j.tics.2019.02.003

Goldberg, A. E. (2006). Constructions at work: The nature of generalization in language. Oxford University Press.

Goldberg, A. E. (2019). Explain Me This: Creativity, Competition, and the Partial Productivity of Constructions. Princeton University Press.

Grice, H. P. (1975). Logic and conversation. In P. Cole \& J. Morgan (Eds.), Syntax and Semantics, vol. 3 (pp. 41-58). Academic Press.

Gundel, J. K., Hedberg, N., \& Zacharski, R. (1993). Cognitive status and the form of referring expressions in discourse. Language, 69(2), 274-307. 
Heller, D., Gorman, K. S., \& Tanenhaus, K. (2012). To name or to describe: shared knowledge affects referential form. Topics in Cognitive Science, 4, 290-305. https://doi.org/10.1111/j.1756-8765.2012.01182.x

Isaacs, E. A., \& Clark, H. H. (1987). References in conversation between experts and novices. Journal of Experimental Psychology, 116(1), 26-37.

Jaeger, T. F. (2010). Redundancy and reduction: Speakers manage syntactic information density. Cognitive Psychology, 61(1), 23-62. https://doi.org/10.1016/j.cogpsych.2010.02.002

Jaeger, T. F., \& Buz, E. (2017). Signal Reduction and Linguistic Encoding. In E. Fernández \& H. Cairns (Eds.), The Handbook of Psycholinguistics (pp. 38-81). John Wiley \& Sons.

Juola, P. (2008). Assessing linguistic complexity. In M. Miestamo, K. Sinnemäki, \& F. Karlsson (Eds.), Language complexity: Typology, contact, change (pp. 89-107). Benjamins. https://doi.org/10.1075/slcs.94.07juo

Koplenig, A., Meyer, P., Wolfer, S., \& Mu, C. (2017). The statistical trade-off between word order and word structure - Large-scale evidence for the principle of least effort. PLoS ONE, 12(3). https://doi.org/10.7910/DVN/8KHoGB

Kravtchenko, E. (2014). Predictability and syntactic production: Evidence from subject omission in Russian. Proceedings of the 36th Annual Conference of the Cognitive Science Society, 785-790. http://people.ucsc.edu/ ekravtch/Kravtchenko_CogSci2014.pdf

Küntay, A. C., \& Slobin, D. I. (1996). Listening to a Turkish mother: some puzzles for acquisition. In D. I. Slobin, J. Gerhardt, A. Kyratzis, \& J. Guo (Eds.), Social interaction, social context, and language: essays in honor of Susan Ervin-Tripp (pp. 265-286). Lawrence Erlbaum Associates.

Kurumada, C., \& Jaeger, T. F. (2015). Communicative efficiency in language production: optional case-marking in Japanese. Journal of Memory and Language, 83, 152-178. https://doi.org/10.1016/j.jml.2015.03.003 
Levy, R., \& Jaeger, T. F. (2007). Speakers optimize information density through syntactic reduction. In B. Schlökopf, J. Platt, \& T. Hoffman (Eds.), Advances in neural information processing systems (NIPS) 19 (pp. 849-856). MIT Press.

Lieven, E., Behrens, H., Speares, J., \& Tomasello, M. (2003). Early syntactic creativity: A usagebased approach. Journal of Child Language, 3o(2), 333-370. https://doi.org/10.1017/So305000903005592

Lieven, E., Salomo, D., \& Tomasello, M. (2009). Two-year-old children's production of multiword utterances: A usage-based analysis. Cognitive Linguistics, 2O(3), 481-507. https://doi.org/10.1515/COGL.2009.022

Linzen, T., \& Jaeger, T. F. (2015). Uncertainty and Expectation in Sentence Processing: Evidence From Subcategorization Distributions. Cognitive Science, n/a-n/a. https://doi.org/10.1111/cogs.12274

Lockridge, C. B., \& Brennan, S. E. (2002). Addressees' needs influence speakers' early syntactic choices. Psychonomic Bulletin \& Review, 9(3), 550-557.

Lombard, E. (1911). Le signe de l'elevation de la voix. Annales de Maladies de L'oreille et Du Larynx, 37, 101-119.

Loy, J. E., \& Smith, K. (2020). Syntactic adaptation may depend on perceived linguistic knowledge: Native English speakers differentially adapt to native and nonnative confederates in dialogue. https://doi.org/10.31234/osf.io/pu2qa

Lupyan, G. (2019). Larger languages have higher entropy. Interaction and the Evolution of Linguistic Complexity.

Lupyan, G., \& Dale, R. (2010). Language structure is partly determined by social structure. PLoS ONE, 5(1). https://doi.org/10.1371/journal.pone.ooo8559

MacWhinney, B. (2000). The CHILDES-project: Tools for analyzing talk. Lawrence Erlbaum. Mahowald, K., Fedorenko, E., Piantadosi, S. T., \& Gibson, E. (2013). Info/information theory: Speakers choose shorter words in predictive contexts. Cognition, 126(2), 313-318. 
https://doi.org/10.1016/j.cognition.2012.09.010

McCauley, S. M., \& Christiansen, M. H. (2014). Acquiring formulaic language: A computational model. The Mental Lexicon, 9(3), 419-436. https://doi.org/10.1075/ml.9.3.03mcc

Newman, R. S., Rowe, M. L., \& Bernstein Ratner, N. (2016). Input and uptake at 7 months predicts toddler vocabulary: The role of child-directed speech and infant processing skills in language development. Journal of Child Language, 43(5), 1158-1173. https://doi.org/10.1017/So305000915000446

Pate, J. K., \& Goldwater, S. (2015). Talkers account for listener and channel characteristics to communicate efficiently. Journal of Memory and Language, 78, 1-17.

Piantadosi, S. T., Tily, H., \& Gibson, E. (2011a). The communicative function of ambiguity in language. Cognition, 122(3), 280-291. https://doi.org/10.1016/j.cognition.2011.10.004

Piantadosi, S. T., Tily, H., \& Gibson, E. (2011b). Word lengths are optimized for efficient communication. Proceedings of the National Academy of Sciences of the United States of America, 108(9), 3526-3529. https://doi.org/10.1073/pnas.1012551108

R Core Team. (2019). R: A Language and Environment for Statistical Computing. R Foundation for Statistical Computing.

Reali, F., \& Christiansen, M. H. (2007). Word chunk frequencies affect the processing of pronominal object-relative clauses. Quarterly Journal of Experimental Psychology, 6o(2), 161-170. https://doi.org/10.1080/17470210600971469

Roche, J. M., Paxton, A., Ibarra, A., \& Tanenhaus, M. K. (2013). From minor mishap to major catastrophe: lexical choice in miscommunication. In M. Knauff, M. Pauen, N. Sebanz, \& I. Wachsmuth (Eds.), Proceedings of the Annual Meeting of the Cognitive Science Society (pp. 3303-3308).

Sanchez, A., Meylan, S. C., Braginsky, M., Macdonald, K. E., Yurovsky, D., \& Frank, M. C. (2019). childes-db: a flexible and reproducible interface to the child language data exchange system. Behavior Research Methods, 51, 1928-1941. 
Schwab, J. F., \& Lew-Williams, C. (2016a). Language learning , socioeconomic status , and childdirected speech. Wiley Interdisciplinary Reviews: Cognitive Science, 7(4), 264-275. https://doi.org/10.1002/wcs.1393

Schwab, J. F., \& Lew-Williams, C. (2016b). Repetition across successive sentences facilitates young children's word learning. Developmental Psychology, 52(6), 879-886.

Shannon, C. E. (1948). A Mathematical Theory of Communication. The Bell System Technical Journal, 27, 379-423.

Siegelman, N., \& Arnon, I. (2015). The advantage of starting big: Learning from unsegmented input facilitates mastery of grammatical gender in an artificial language. Journal of Memory and Language, 85, 60-75. https://doi.org/10.1016/j.jml.2015.07.003

Siegelman, N., Rueckl, J. G., Steacy, L. M., Frost, S. J., van den Bunt, M., Zevin, J. D., Seidenberg, M. S., Pugh, K. R., Compton, D. L., \& Morris, R. D. (2020). Individual differences in learning the regularities between orthography, phonology and semantics predict early reading skills. Journal of Memory and Language, 114, 104145. https://doi.org/10.1016/j.jml.2020.104145

Skarabela, B., Ota, M., O’Connor, R., \& Arnon, I. (2021). 'Clap your hands' or ‘take your hands’? One-year-olds distinguish between frequent and infrequent multiword phrases. Cognition, 211, 104612. https://doi.org/10.1016/j.cognition.2021.104612

Snow, C. E. (1972). Mothers' speech to children learning language. Child Development, 43(2), 549-565.

Soderstrom, M. (2007). Beyond babytalk: re-evaluating the nature and content of speech input to preverbal infants. Developmental Review, 27, 501-532. https://doi.org/10.1016/j.dr.2007.06.002

Stoll, S., Abbot-Smith, K., \& Lieven, E. (2009). Lexically restricted utterances in Russian, German, and English child-directed speech. Cognitive Science, 33, 75-103. https://doi.org/10.1111/j.1551-6709.2008.01004.x 
Summers, W. Van, Pisoni, D. B., Bernacki, R. H., Pedlow, R. I., \& Stokes, Michael, A. (1988). Effects of noise on speech production: acoustic and perceptual analyses. The Journal of the Acoustical Society of America, 84(3), 917-928.

Tal, S., \& Arnon, I. (2018). SES effects on the use of variation sets in child-directed speech. Journal of Child Language, 45(6), 1423-1438. https://doi.org/10.1017/So305000918000223

Tal, S., Grossman, E., Rohde, H., \& Arnon, I. (2023). Speakers use more redundant references with language learners: evidence for communicatively-efficient referential choice. Journal of Memory and Language, 128, 104378. https://doi.org/10.1016/j.jml.2022.104378

Tily, H., \& Piantadosi, S. (2009). Refer efficiently: Use less informative expressions for more predictable meanings. Proceedings of the Workshop on the Production of Referring Expressions: Bridging the Gap between Computational and Empirical Approaches to Reference.

Tippenhauer, N., Fourakis, E. R., Watson, D. G., \& Lew-Williams, C. (2020). The scope of audience design in child-directed speech: parents' tailoring of word lengths for adult versus child listeners. Journal of Experimental Psychology: Learning, Memory, and Cognition, 46(11), 2163-2178. https://doi.org/10.1017/CBO9781107415324.004

Tomasello, M. (2003). Constructing a language: A Usage-Based theory of language acquisition. Harvard University Press.

Uther, M., Knoll, M. A., \& Burnham, D. (2007). Do you speak E-NG-L-I-SH ? A comparison of foreigner- and infant-directed speech. Speech Communication, 49, 2-7. https://doi.org/10.1016/j.specom.2006.10.003

Waterfall, H. (2006). A little change is a good thing: feature theory, language acquisition and variation sets (Doctoral dissertation). University of Chicago.

Yurovsky, D., Doyle, G., \& Frank, M. C. (2016). Linguistic input is tuned to children's developmental level. Proceedings of the 38th Annual Meeting of the Cognitive Science 
Society, 2094-2098.

Zaslavsky, N., Kemp, C., Regier, T., \& Tishby, N. (2018). Efficient compression in color naming and its evolution. Proceedings of the National Academy of Sciences, 115(31), 7937-7942. https://doi.org/10.1073/pnas.1800521115

Zhao, Y., \& Jurafsky, D. (2009). The effect of lexical frequency and Lombard reflex on tone hyperarticulation. Journal of Phonetics, 37, 231-247.

https://doi.org/10.1016/j.wocn.2009.03.002

Zipf, G. K. (1949). Human behavior and the principle of least effort: An introduction to human ecology. Addison-Wesley Press. 